J. Phys. E: Sci. Instrum., Vol. 17, 1984. Printed in Great Britain

\section{A simple and inexpensive laser power meter}

\author{
A I Ferguson \\ Physics Department, The University, Southampton S09 5NH, \\ $\mathrm{UK}$
}

\section{Received 18 August 1983, in final form 15 September 1983}

Abstract. The construction of a simple and inexpensive laser power meter is described. The laser power is monitored using a calorimeter sensor which is based on the use of commercial platinum resistors. In this design, the use of volume absorbing glass enables the meter to withstand peak power densities in the region of $100 \mathrm{MW} \mathrm{cm}^{-2}$ and average powers in excess of $10 \mathrm{~W}$ at $1.06 \mu \mathrm{m}$. Average powers down to less than $1 \mathrm{~mW}$ can also be readily measured.

\section{Introduction}

The widespread use of Q-switched Nd:YAG lasers of high repetition rate has brought about a need for a laser power meter which will measure average powers of several watts and will withstand energy densities in excess of $1 \mathrm{~J} \mathrm{~cm}^{-2}$ in a $10 \mathrm{~ns}$ pulse. A common solution to the damage problems which this creates has been the use of volume absorbers which are thermally bonded to a metallic substrate (Gunn 1973). The rise in temperature (between this substrate and a heat sink) caused by the absorption of laser radiation is measured using a thermopile. Many types of laser power meters have been described in the literature including photodiodes, pyroelectric detectors, thermopiles and calorimeters (Gunn 1974, McCormack 1982, Shaw 1981, Franzen and Schmidt 1976). In the design described here the temperature rise due to absorbed laser radiation is measured by monitoring the change in resistance of a commercial platinum resistor. These resistors are readily available, inexpensive and are very robust.

\section{Design}

A schematic diagram of the power meter is shown in figure 1. The power meter head consists of a massive, solid aluminium alloy base which acts as a heat sink. The sensing element consists of two platinum resistors (Matthey Printed Products Ltd, Type 100S25). These resistors are a thin film of platinum coated onto an alumina substrate. The substrate is $25.4 \mathrm{~mm}$ square by $0.8 \mathrm{~mm}$ thick. The sensor is thermally bonded to the heat sink using a zinc-loaded compound with the detector track away from the heat sink. A second sensor is thermally contacted to the first substrate again using a thin layer of thermally conducting compound. The detector tracks in this case are also away from the heat sink. Finally, the absorber is bonded to the second sensor using thermally conducting compound. The absorber may be a surface or volume absorber but for this particular design we describe a volume absorber. The volume absorber is a neutrally coloured glass filter (Schott NG1) of $1 \mathrm{~mm}$ thickness and $25.4 \mathrm{~mm}$ square. The internal absorption of this glass is greater than $99 \%$ over the near-ultraviolet and visible regions of the spectrum. Absorption takes place in the bulk of the medium over the range of about $400 \mathrm{~nm}$ to $1.2 \mu \mathrm{m}$. Outside this range the absorption coefficient is so large that the absorption is in the surface of the glass. All three components, the two sensors and the filter glass, are secured at their corners by a small amount of epoxy resin cement.

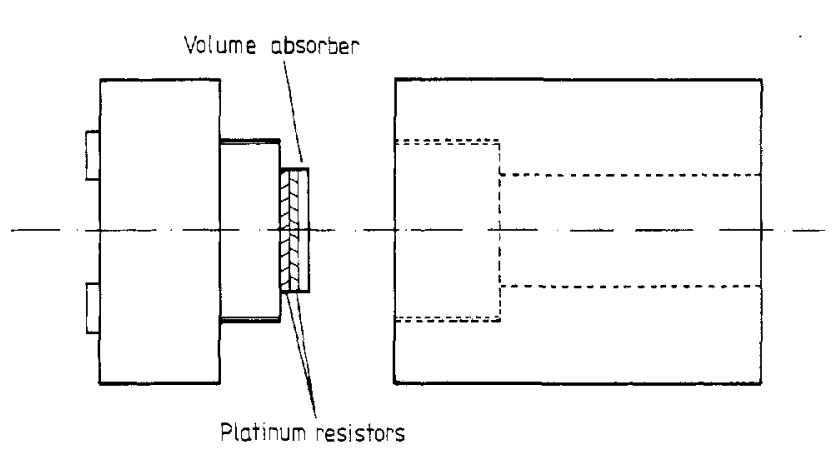

Figure 1. Schematic of the power meter head. The two platinum resistors and volume absorber are thermally contacted to the massive alloy heat sink. A cylinder which provides thermal shielding is also shown.

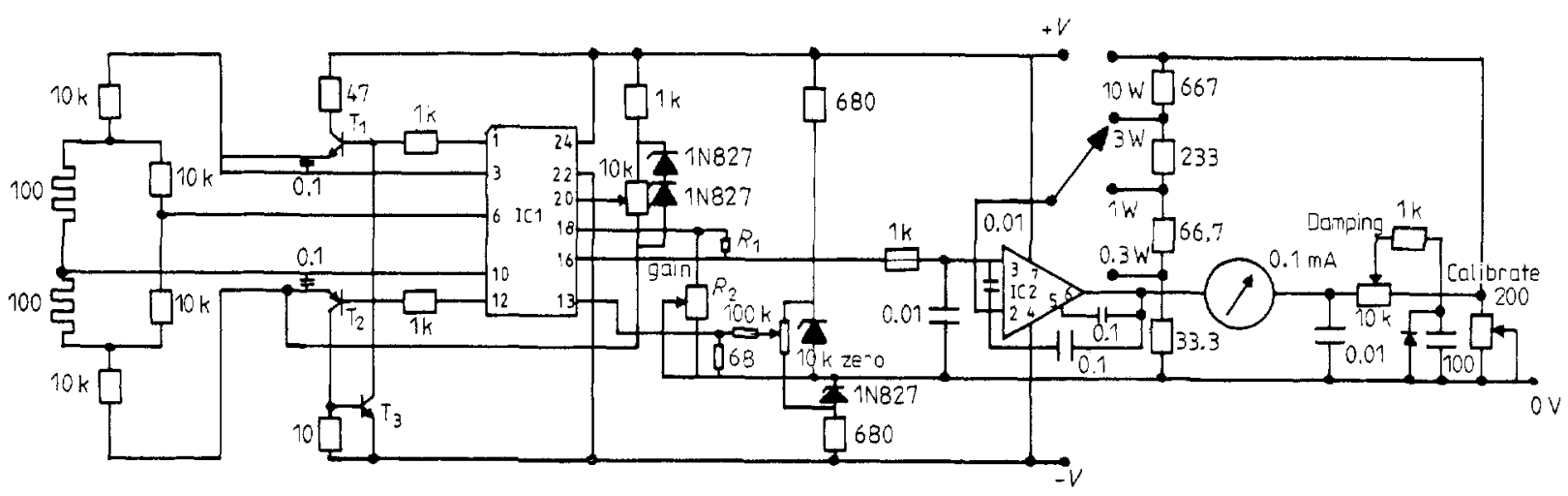

Figure 2. Circuit diagram of the amplifier used in the power meter. The resistors $R_{1}$ and $R_{2}$ set the gain of the bridge amplifier (gain $=1+R_{1} / R_{2}$ ). Typical values for $R_{1}$ and $R_{2}$ are $100 \mathrm{k} \Omega$ and $20 \mathrm{k} \Omega$ respectively. Zero damping and calibration adjustments are incorporated into the design. $\mathrm{T}_{1}, \mathrm{BD} 135 ; \mathrm{T}_{2}, \mathrm{BD} 136 ; \mathrm{T}_{3}, \mathrm{BC} 108$, IC1, SGA301; IC2, Op-07. 
The electrical connections to the platinum resistors are taken to the back of the heat sink using $4 \mathrm{~mm}$ sockets. Thermal shielding and further heat sinking is provided by a massive cylinder of aluminium alloy which is screwed to the base. A hole of $23 \mathrm{~mm}$ diameter along the axis of the cylinder allows the laser to impinge on the volume absorber.

The heating of the volume absorber due to the absorption of incident laser radiation is detected by monitoring the difference in resistance between the platinum resistors. This difference is an indication of the absorbed laser power. The bridge circuit used to measure this difference is shown in figure 2. The bridge amplifier is an integrated device providing suitable positive and negative stabilised supply voltages for the bridge (CIL Electronics Ltd, Type SGA301). The resistors in series with the bridge are matched to within $0.1 \%$ and are used to limit the current through the sensors. In addition, their resistance is large compared to the nominal resistance of the platinum sensors and so they are used to maintain an almost constant current as the resistance of the sensors changes.

The resistors $R_{1}$ and $R_{2}$ are chosen to set the gain of the amplifier for approximate calibration. Typical values for $R_{1}$ and $R_{2}$ are $100 \mathrm{k} \Omega$ and $20 \mathrm{k} \Omega$ respectively. The output from the bridge amplifier is then fed to a switched-gain operational amplifer which acts as a voltage-to-current converter. The final calibration of the instrument can be adjusted using a variable resistor.

The thermal response time of the power meter head is in the region of $5 \mathrm{~s}$. This is an uncomfortably long time when the power meter is being used to monitor the laser power while adjustments are made. The effective time constant of the power meter head can be greatly reduced by incorporating a circuit which anticipates the equilibrium setting of the meter as the power is changed. By adjusting the setting of the damping resistor it is possible to reduce the effective time constant of the meter to less than one second.

We have investigated the uniformity of response of the meter over its surface area by mounting the head on a horizontal and vertical stage. We have found that the response is uniform to within $2 \%$ over the central $20 \mathrm{~mm}$ diameter. The zero drift has also been checked and found to be less than $3 \mathrm{~mW}$ over a period of $1 \mathrm{~h}$ under normal laboratory conditions. The meter has been calibrated by comparing the readings given by it and by two commercially available power meters (Coherent 210 and Scientech 38-1010). It has been found that all three meters are in agreement to within $5 \%$ over powers ranging from $10 \mathrm{~mW}$ to $10 \mathrm{~W}$ using several pulsed and continuous-wave lasers.

\section{Conclusions}

We have described a simple and reliable laser power meter which can be constructed from readily available commercial components. It is expected that the long-term stability of the platinum resistors should be excellent and that calibration should be maintained for long periods. The design lends itself to the inclusion of a substitution heater for absolute calibration (Gunn 1974). These ideas are being investigated in our laboratory at present.

\section{Acknowledgments}

The financial support from SERC is gratefully acknowledged. The author wishes to thank Dr P C Lanchester for many illuminating discussions.

\section{References}

Gunn S R 1973 Calorimetric measurements of laser energy and power

J. Phys. E: Sci. Instrum. 6 105-14
Gunn S R 1974 Volume-absorbing calorimeters for high-power laser pulses

Rev. Sci. Instrum. 45 936-43

McCormack M 1982 Laser power meters

Electro-Optical Systems Design 14 35-46

Shaw R W 1981 Measuring pulse energies optoacoustically Laser Focus 17 69-72

Franzen D L and Schmidt L B 1976 Absolute reference calorimeter for measuring high power laser pulses

Appl. Opt. $153115-22$ 